\title{
Vehicle Cruise: Improved Fuel Economy by Periodic Control $^{* \dagger}$
}

\author{
ELMER G. GILBERT $\ddagger$
}

\begin{abstract}
A simple dynamic model, formulated for vehicles in cruise, shows that time-dependent periodic control gives better fuel economy than optimum steady-state control under appropriate conditions on the drag and fuel-consumption functions.
\end{abstract}

\begin{abstract}
Summary-It is shown that time-dependent periodic control can improve the fuel economy of vehicles in cruise. The time-dependent controls considered are relaxed steady-state (RSS) control, quasi-steady-state (QSS) control and quasi-relaxed steady-state (QRSS) control. Examples are given which show that QRSS control may give better performance than either RSS or QSS control. Properties of optimal cost functions, dependent on the minimum required average speed, are derived. The possibility or impossibility of improved performance through the use of QRSS, QSS and RSS control is investigated in terms of assumptions on the vehicle drag and fuelconsumption functions.
\end{abstract}

\section{INTRODUCTION}

IT is traditional to operate many dynamic processes in an optimal steady-state mode where the controls and system state are constant and chosen to extremize a performance function subject to process constraints and equilibrium equations. While this approach has considerable intuitive appeal it is not always best. There are many examples, taken mostly from the field of chemical engineering [1], where time-dependent periodic control of the process yields improved performance. The problem of vehicle cruise presented here shows that periodic control can, under appropriate conditions, yield better fuel economy than conventional optimal steady-state control. In particular, both relaxed steady-state control and quasi-steady-state control can do better. The problem also illustrates, for the first time, that quasi-relaxed steady-state control [2] may be better than either relaxed steady-state control or quasi-steady-state control.

\footnotetext{
* Received 17 May 1975; revised 22 September 1975. The original version of this paper was not presented at any IFAC meeting. It was recommended for publication in revised form by associate editor $P$. Dorato.

t Most of the results in this paper were presented at the 1975 Conference on Information Sciences and Systems, The Johns Hopkins University, Baltimore, Maryland, U.S.A., April 1975.

† Department of Aerospace Engineering, Program in Computer, Information and Control Engineering, The University of Michigan, Ann Arbor, Michigan 48104, U.S.A.
}

The vehicle cruise problem is formulated as follows. The performance function

$$
\begin{aligned}
J(T(\cdot), V(\cdot), \tau) & =V_{\mathrm{avg}}\left(F_{\mathrm{avg}}\right)^{-1} \\
& =\text { specific range }
\end{aligned}
$$

depends on the thrust $T(\cdot)$ (measurable on $[0, \tau)$ ), the speed $V(\cdot)$, and the period $\tau>0$ which satisfy the following constraints:

$$
\begin{aligned}
\dot{V} & =-D(V)+T(t), \quad V(0)=V(\tau) \geqslant 0, \\
0 & \leqslant T(t) \leqslant 1, \quad \text { a.a. } t \in[0, \tau], \\
F_{\text {avg }} & =\frac{1}{\tau} \int_{0}^{\tau} F(T(t)) \mathrm{d} t=\text { average fuel rate, } \\
V_{\text {avg }} & =\frac{1}{\tau} \int_{0}^{\tau} V(t) \mathrm{d} t=\text { average speed, } \\
V_{\text {avg }} & \geqslant V_{\min } \geqslant 0 .
\end{aligned}
$$

The condition $V(0)=V(\tau)$ assures that both $V(\cdot)$ and $T(\cdot)$ are periodic when the domain of these functions is extended to $(-\infty,+\infty)$ by

$$
V(t+\tau)=V(t) \text { and } T(t+\tau)=T(t) .
$$

It is assumed that: the drag function $D(\cdot)$ : $[0,+\infty) \rightarrow[0,+\infty)$ is Lipschitz continuous and the equation $D(\mathrm{~V})=1$ has a unique solution $V=V_{\max }$. Thus $V_{\max }$ is the maximum steady-state speed. Moreover, it is not difficult to show that $V(\cdot)$ satisfies (2) and (3) only if $0 \leqslant V(t) \leqslant V_{\max }, 0 \leqslant t \leqslant \tau$. To assure that the speed constraint (6) can be satisfied it is required that $0 \leqslant V_{\min } \leqslant V_{\max }$. The fuelconsumption function $F(\cdot):[0,1] \rightarrow(0,+\infty)$ is assumed to be lower semi-continuous and nondecreasing $\left(F\left(T_{2}\right) \geqslant F\left(T_{1}\right), 0 \leqslant T_{1} \leqslant T_{2} \leqslant 1\right)$. Positive jumps in $F(T)$ permit the modelling of multi-engine propulsion systems where the fuel flow rate may jump discontinuously with the turning-on of additional engines. The requirement of lower semicontinuity is for mathematical convenience; it implies the existence of appropriate minima. In any case the assumptions on $D(\cdot)$ and $F(\cdot)$ are not 
physically unreasonable and they allow for a rich supply of interesting examples. Inspection shows that the specific range is bounded:

$$
0 \leqslant J \leqslant V_{\max }(F(0))^{-1} .
$$

The maximization of $J(T(\cdot), V(\cdot), \tau)$ subject to the constraints is an optimal periodic control (OPC) problem [1-3]. Solutions of OPC may be sought through the application of necessary conditions $[3,4]$, but in what follows the main interest is in specialized optimal steady-state controls which simplify the analysis and yield, at least in an approximate sense, $T(\cdot), V(\cdot)$, and $\tau$ which still satisfy (2-6). Hopefully, the study of these special controls helps in understanding the mechanisms by which time-dependent control improves performance. The optimal values of $J$ for these steady-state controls also shed light on the solution of OPC since they never exceed $J^{*}$, the optimal $J$ for OPC.

\section{THE FOUR STEADY-STATE PROBLEMS}

The optimal steady-state (SS) problem is obtained from OPC by restricting $T(\cdot)$ and $V(\cdot)$ to be constant $(\mathrm{T}(t) \equiv T, V(t) \equiv V)$. In this case the value of $\tau$ makes no difference and (1-6) reduce to

$$
\begin{aligned}
J=V(F(T))^{-1}, & D(V)=T, \\
& 0 \leqslant T \leqslant 1, \quad V_{\min } \leqslant V .
\end{aligned}
$$

Using $T=D(V)$ to eliminate $T$, gives

$$
J=V\left(f_{\mathrm{SS}}(V)\right)^{-1}, \quad V_{\min } \leqslant V \leqslant V_{\max },
$$

where

$$
f_{\mathrm{SS}}(V)=F(D(V)) .
$$

The maximum of $J$ exists (because $f_{\mathrm{sg}}(\cdot)$ is lower semi-continuous and $J$ is bounded from above) and depends on the value of $V_{\min }$. Thus for $V_{\min } \in[0$, $\left.V_{\max }\right]$

$$
\begin{aligned}
J_{\mathrm{Ss}}{ }^{*}\left(V_{\min }\right)= & \max V\left(f_{\mathrm{SS}}(V)\right)^{-1} \\
& \text { subject to } V \in\left[V_{\min }, V_{\max }\right]
\end{aligned}
$$

is defined and characterizes the solution of the optimal SS problem for all $V_{\min }$. If $J^{*}\left(V_{\min }\right)$ is the optimum cost for OPC (let $J^{*}\left(V_{\min }\right)$ be the supremum if the maximum does not exist) then $J^{*}(\cdot) \geqslant J_{\mathrm{SS}}{ }^{*}(\cdot)$. Here and in what follows the notation $f_{1}(\cdot) \geqslant f_{2}(\cdot)$ is used if and only if $f_{1}(V) \geqslant$ $f_{2}(V)$ for all $V \in\left[0, V_{\max }\right]$. The three remaining steady-state problems correspond to limiting forms of time-dependent periodic control.

Relaxed steady-state (RSS) controls are obtained by defining

$$
T(t)= \begin{cases}T_{1}, & 0 \leqslant t<\lambda \tau \\ T_{2}, & \lambda \tau \leqslant t<\tau,\end{cases}
$$

where $0 \leqslant \lambda \leqslant 1$, and letting $\tau \rightarrow 0$. The periodicity condition and differential equation (2) then require that $-\int_{0}^{\tau} D(V(t)) \mathrm{d} t+\tau\left(\lambda T_{1}+(1-\lambda) T_{2}\right)=0$. As $\tau \rightarrow 0,\left(V(t)-V_{\text {avg }}\right)$ tends to zero on $[0, \tau]$ and thus $D\left(V_{\mathrm{avg}}\right) \rightarrow \lambda T_{1}+(1-\lambda) T_{2}$. Since

$$
F_{\mathrm{avg}}=\lambda F\left(T_{1}\right)+(1-\lambda) F\left(T_{2}\right)
$$

this leads to the following RSS optimization problem:

$$
\begin{aligned}
J= & V\left(\lambda F\left(T_{1}\right)+(1-\lambda) F\left(T_{2}\right)\right)^{-1}, \\
D(V)= & T_{1} \lambda+(1-\lambda) T_{2}, \\
0 \leqslant & T_{1} \leqslant 1, \quad 0 \leqslant T_{2} \leqslant 1, \quad 0 \leqslant \lambda \leqslant 1, \\
& V_{\min } \leqslant V,
\end{aligned}
$$

where for simplicity $V_{\text {avg }}$ has been replaced by $V$. Define the convex hull of $F(\cdot),(\operatorname{conv} F)(\cdot)$, by

$$
\begin{array}{r}
(\operatorname{conv} F)(T)=\min \lambda F\left(T_{1}\right)+(1-\lambda) F\left(T_{2}\right) \\
\text { subject to } \lambda T_{1}+(1-\lambda) T_{2}=T \\
\text { and } \lambda, T_{1}, T_{2} \in[0,1] .
\end{array}
$$

This function is the 'largest' convex function which is a lower bound for $F(\cdot)$ [5]. It follows that maximizing $J$ in (12) corresponds to maximizing $J$ in

$$
J=V\left(f_{\mathrm{RSS}}(V)\right)^{-1}, \quad V_{\min } \leqslant V \leqslant V_{\max },
$$

where

$$
f_{\mathrm{RSS}}(V)=(\operatorname{conv} F)(D(V)) .
$$

Since $(\operatorname{conv} F)(\cdot)$ is convex and therefore continuous, $f_{\mathrm{RSS}}(\cdot)$ is continuous, though not necessarily convex. This and $f_{\mathrm{RSS}}(V) \geqslant F(0)>0$ implies $J$ has a maximum. Thus as in (10) define

$$
\begin{aligned}
J_{\mathrm{RSS}}{ }^{*}\left(V_{\min }\right)= & \max V\left(f_{\mathrm{RSS}}(V)\right)^{-1} \\
& \text { subject to } V \in\left[V_{\min }, V_{\max }\right] .
\end{aligned}
$$

Since $f_{\mathrm{RSS}}(\cdot) \leqslant f_{\mathrm{SS}}(\cdot)$ it follows that

$$
J^{*}(\cdot) \geqslant J_{\mathrm{Rss}}{ }^{*}(\cdot) \geqslant J_{\mathrm{ss}}{ }^{*}(\cdot) .
$$

Quasi-steady-state (QSS) controls are obtained by switching from one constant speed $V_{1}$ to another constant speed $V_{2}$ and letting the time between switches become large. Assume $0<V_{1} \leqslant V_{2}<V_{\max }$ and define

$$
T(t)=\left\{\begin{array}{l}
0, \quad 0 \leqslant t<\Delta t_{1}, \\
D\left(V_{1}\right), \quad \Delta t_{1} \leqslant t<\Delta t_{1}+\lambda \bar{\tau}, \\
+1, \quad \Delta t_{1}+\lambda \bar{\tau} \leqslant t<\Delta t_{1}+\lambda \bar{\tau}+\Delta t_{2}, \\
D\left(V_{2}\right), \quad \Delta t_{1}+\lambda \bar{\tau}+\Delta t_{2} \leqslant t<\tau,
\end{array}\right.
$$

where $\tau=\Delta t_{1}+\Delta t_{2}+\bar{\tau}, 0 \leqslant \lambda \leqslant 1, \Delta t_{1}$ is the time (finite because $V_{1}>0$ ) required in (2) to go from $V=V_{2}$ to $V=V_{1}$ with $T(t) \equiv 0, \Delta t_{2}$ is the time (finite because $V_{2}<1$ ) required in (2) to go from 
$V=V_{1}$ to $V=V_{2}$ with $T(t) \equiv 1$. As $\bar{\tau} \rightarrow+\infty$ it is clear that

$$
\begin{aligned}
F_{\mathrm{avg}} \rightarrow \lambda F\left(D\left(V_{1}\right)\right) & +(1-\lambda) F\left(D\left(V_{2}\right)\right)=\lambda f_{\mathrm{SS}}\left(V_{1}\right) \\
& +(1-\lambda) f_{\mathrm{SS}}\left(V_{2}\right)
\end{aligned}
$$

and $V_{\mathrm{avg}} \rightarrow \lambda V_{1}+(1-\lambda) V_{2}$. If either $V_{1}=0$ or $V_{2}=V_{\max }$ the result is the same but the argument is a little more complicated because $V_{1}$ or $V_{2}$ can only be approached. Thus QSS controls lead to the following optimization problem:

$$
\begin{gathered}
J=\left(\lambda V_{1}+(1-\lambda) V_{2}\right)\left(\lambda f_{\mathrm{SS}}\left(V_{1}\right)\right. \\
\left.+(1-\lambda) f_{\mathrm{SS}}\left(V_{2}\right)\right)^{-1}, \\
0 \leqslant V_{1} \leqslant V_{2} \leqslant 1, \quad 0 \leqslant \lambda \leqslant 1 .
\end{gathered}
$$

Define

$$
\begin{aligned}
f_{\mathrm{QSS}}(V)= & \left(\operatorname{conv} f_{\mathrm{SS}}\right)(V)=\min \lambda f_{\mathrm{SS}}\left(V_{1}\right) \\
& +(1-\lambda) f_{\mathrm{SS}}\left(V_{2}\right) \quad \text { subject to } \lambda V_{1} \\
& +(1-\lambda) V_{\mathbf{2}}=V, \quad \lambda \in[0,1] \\
& \text { and } V_{1}, V_{\mathbf{2}} \in\left[0, V_{\max }\right]
\end{aligned}
$$

and the maximizing of $J$ in (18) is equivalent to maximizing $J$ in

$$
J=V\left(f_{\mathrm{QSS}}(V)\right)^{-1}, \quad V_{\min } \leqslant V \leqslant V_{\max } .
$$

Since $f_{\mathrm{QSS}}(\cdot)$ is continuous and bounded away from zero the maximum exists and can be written

$$
\begin{aligned}
J_{\mathrm{QSS}} *\left(V_{\min }\right)= & \max V\left(f_{\mathrm{QSS}}(V)\right)^{-1} \\
& \text { subject to } V \in\left[V_{\min }, V_{\max }\right] .
\end{aligned}
$$

Because of $(19), f_{\mathrm{QSS}}(\cdot) \leqslant f_{\mathrm{SS}}(\cdot)$ and therefore $J^{*}(\cdot) \geqslant J_{\mathrm{QSs}} *(\cdot) \geqslant J_{\mathrm{Ss}} *(\cdot)$.

Finally, it is possible to switch, with the time between switchings becoming large, between two speeds $V_{1}$ and $V_{2}$ where either or both $V_{1}$ and $V_{2}$ are maintained by RSS controls. This is the quasirelaxed steady-state (QRSS) case. Without writing down the details it should be obvious how to proceed. The effective fuel consumption at $V_{\mathrm{i}}$ is $f_{\mathrm{RSS}}\left(V_{\mathrm{i}}\right)$ instead of $f_{\mathrm{SS}}\left(V_{\mathrm{j}}\right)$. Making this substitution in the analysis of the previous paragraph means that $(20)$ is replaced by

$$
J=V\left(f_{\mathrm{QRSS}}(V)\right)^{-1}, \quad V_{\min } \leqslant V \leqslant V_{\max },
$$

where

$$
f_{\text {QRSS }}(V)=\left(\operatorname{conv} f_{\mathrm{RSS}}\right)(V) .
$$

As before the maximum of $J$ in (22) exists and it is possible to define

$$
\begin{aligned}
& J_{\mathrm{QRSS}} *\left(V_{\min }\right)=\max V\left(f_{\mathrm{QRSS}}(V)\right)^{-1} \\
& \text { subject to } V \in\left[V_{\min }, V_{\mathrm{max}}\right] .
\end{aligned}
$$

From (23), $\quad f_{\text {QRSS }}(\cdot) \leqslant f_{\text {RSS }}(\cdot)$ and therefore $J^{*}(\cdot) \geqslant J_{\mathrm{QRSS}^{*}}(\cdot) \geqslant J_{\mathrm{RSs}^{*}}(\cdot) \geqslant J_{\mathrm{SS}}{ }^{*}(\cdot)$. Since $f_{\mathbf{1}}(\cdot) \leqslant$ $f_{2}(\cdot)$ implies $\left(\operatorname{conv} f_{1}\right)(\cdot) \leqslant\left(\operatorname{conv} f_{2}\right)(\cdot)$ it follows from $f_{\mathrm{RSS}}(\cdot) \leqslant f_{\mathrm{SS}}(\cdot)$ that $f_{\mathrm{QRSS}}(\cdot) \leqslant f_{\mathrm{QSS}}(\cdot)$. Thus $J^{*}(\cdot) \geqslant J_{\mathrm{QRSS}} *(\cdot) \geqslant J_{\mathrm{QSS}} *(\cdot) \geqslant J_{\mathrm{Ss}} *(\cdot)$.
Figure 1 illustrates the preceding definitions and results for representative drag and fuel consumption functions. To interpret Fig. 1(c) it helps to
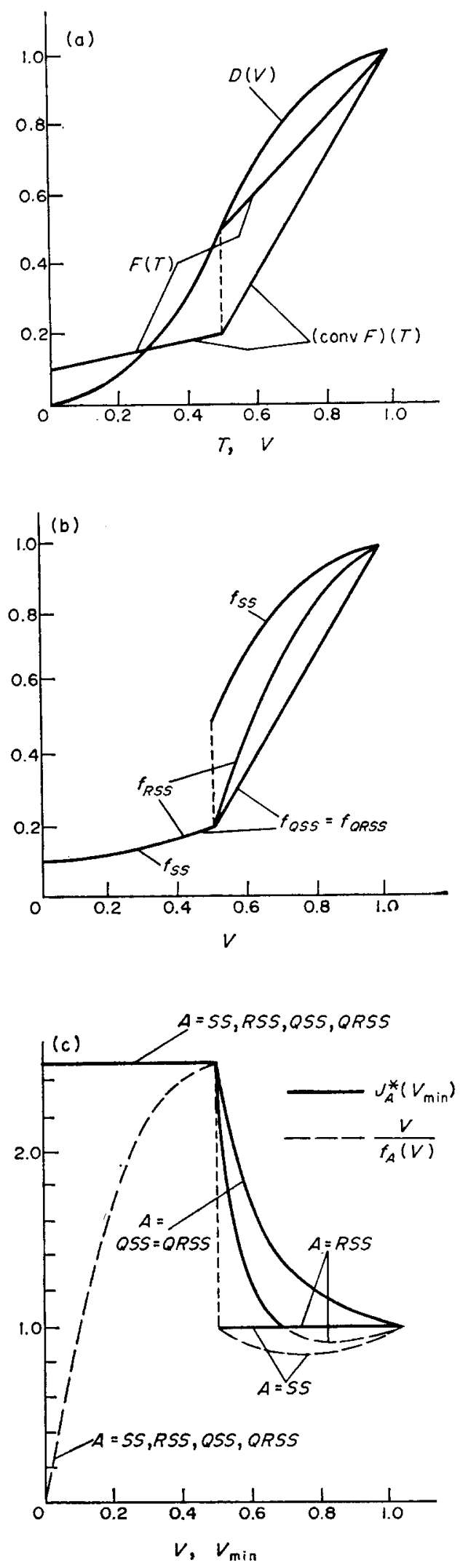

Fio. 1. Example illustrating definitions and results of Section 2 . 
notice in Fig. 1(b) that lines of fixed specific range are of the form $f=J^{-1} V$. Thus lines of least slope correspond to maximum specific range.

\section{EXAMPLES WHERE QRSS CONTROL IS BETTER}

In this section two examples are considered where $J_{\mathrm{QRSS}} *\left(V_{\min }\right)$ is greater than both $J_{\mathrm{Rgs}} *\left(V_{\min }\right)$ and $J_{\mathrm{QSS}} *\left(V_{\mathrm{min}}\right)$. For each example the functions $f_{\mathrm{SS}}, f_{\mathrm{RSS}}, f_{\mathrm{QSS}}, f_{\mathrm{QRSS}}, J_{\mathrm{SS}}{ }^{*}, J_{\mathrm{RSS}}{ }^{*}, J_{\mathrm{QSS}} *$ and $J_{\mathrm{QRSS}}{ }^{*}$ are characterized and there is some general discussion. The details of the derivations entail simple analytic geometry and are therefore omitted. It is easy to be convinced that the form of the results is correct by sketching the functions involved.

\section{Example 1}

The drag and fuel-consumption functions are given by

$D(V)=\left\{\begin{array}{l}0 \cdot 1 V+2 \cdot 7 V^{2}-1 \cdot 8 V^{3}, \quad 0 \leqslant V \leqslant 1, \\ 0 \cdot 9+0 \cdot 1 V, \quad 1 \leqslant V\end{array}\right.$

and

$$
F(T)= \begin{cases}0 \cdot 05+1 \cdot 2 T, & 0 \leqslant T \leqslant 0 \cdot 5, \\ 0 \cdot 25+0 \cdot 8 T, & 0.5 \leqslant T \leqslant 1 .\end{cases}
$$

Since $D(1)=1$ and $D(0 \cdot 5)=0 \cdot 5, V_{\max }=1$ and

$$
f_{\mathrm{Ss}}(V)= \begin{cases}0.05+1.2 D(V), & 0 \leqslant V \leqslant 0.5, \\ 0.25+0.8 D(V), & 0.5 \leqslant V \leqslant 1\end{cases}
$$

Note that $f_{\mathrm{SS}}(\cdot)$ is continuous because $F(\cdot)$ is continuous. Since $F(\cdot)$ is concave (conv $F$ ) $(T)=0.05+T<F(T)$ for $0<T<1$. Thus

$$
f_{\mathrm{RSS}}(V)=0.05+D(V)
$$

and $f_{\mathrm{RSS}}(V)<f_{\mathrm{SS}}(V)$ for $0<V<1$. For $0<V<1$ the RSS control switches at 'high' frequency between $T_{1}=1$ and $T_{2}=0$ and $\lambda$ in (11) is determined by $D(V)=\lambda$. The function $f_{\mathrm{SS}}(\cdot)$ is convex on $[0,0 \cdot 5]$ but concave on $[0 \cdot 5,1]$. Thus $f_{\mathrm{QSS}}(V)<$ $f_{\mathrm{SS}}(V)$ for some $V$. In fact

$$
f_{\mathrm{QSS}}(V)=\left\{\begin{array}{c}
0.05+1.2 D(V)=f_{\mathrm{SS}}(V), \\
0 \leqslant V \leqslant V_{1}, \\
1.05+(1.0805 \ldots)(V-1) \\
<f_{\mathrm{SS}}(V), \quad V_{1}<V<1,
\end{array}\right.
$$

where $V_{1}=(0 \cdot 1809 \ldots)$. For $V_{1}<V<1$ 'low' frequency switching between $V_{1}$ and $V_{2}=1$ occurs and $\lambda$ is determined by $V=\lambda V_{1}+1-\lambda$. Inspection also shows that $f_{\mathrm{RSs}}(\cdot)$ is not convex and it turns out that

$$
f_{\mathrm{QRSS}}(V)=\left\{\begin{array}{l}
0 \cdot 05+D(V)=f_{\mathrm{RSs}}(V) \\
0 \leqslant V \leqslant 0 \cdot 25 \\
1 \cdot 05+1 \cdot 1125(V-1)<f_{\mathrm{RSS}}(V) \\
0.25<V<1 .
\end{array}\right.
$$

For $0 \cdot 25<V<1$ there is 'low' frequency switching between $V_{1}=0.25$ and $V_{2}=1$ with $\lambda$ determined by $V=0 \cdot 25 \lambda+1-\lambda=1-0.75 \lambda$. The speed $V_{1}$ is maintained by 'high' frequency switching between $T_{1}=1$ and $T_{2}=0$ with $\lambda_{\mathrm{R}}$, the fraction of time spent at $T(t)=T_{1}$, being determined by $\lambda_{\mathrm{R}}=$ $D(0 \cdot 25)=0 \cdot 165625$. The speed $V_{2}=1$ is maintained by a constant $T(t) \equiv+1$. Clearly, $f_{\mathrm{QRSS}}(V)$ $<f_{\mathrm{QSS}}(V)$ for $0<V<1$. In the neighborhood of $V=0, f_{\mathrm{RSS}}(V)<f_{\mathrm{QSS}}(V)$; in the neighborhood of $V=1, \quad f_{\mathrm{QSS}}(V)<f_{\mathrm{RSS}}(V) . \quad$ Therefore neither $f_{\mathrm{RSS}}(\cdot) \leqslant f_{\mathrm{QSS}}(\cdot)$ nor $f_{\mathrm{QSS}}(\cdot) \leqslant f_{\mathrm{RSS}}(\cdot)$ is possible.

The implications of the above results with respect to $J_{\mathrm{SS}} *(\cdot), J_{\mathrm{RSS}}{ }^{*}(\cdot), J_{\mathrm{QSS}}{ }^{*}(\cdot)$ and $J_{\mathrm{QRSS}} *(\cdot)$ is shown in Fig. 2. The values of $a, b, c, d$ and $e$ are: $(0 \cdot 1374 \ldots),(0 \cdot 1524 \ldots),(0 \cdot 2897 \ldots),(0 \cdot 3833 \ldots)$ and $(0.4363 \ldots)$. Each of the four types of steadystate control apply for $V_{\min }$ in certain intervals: QRSS for $(0 \cdot 25,1)$, QSS for $(0 \cdot 1809 \ldots, 1)$, RSS for $[0, e)$ and $\mathrm{SS}$ for $[0,1]$. For $V_{\min } \in[0, a]$, SS control requires $T=D(a)$; for $V_{\min } \in[0, b]$, RSS control requires $\lambda=D(b)$; for $V_{\min } \in[c, 1]$, SS control requires $T=1$. Neither $J_{\mathrm{RSS}} *(\cdot) \leqslant J_{\mathrm{QSS}} *(\cdot)$ nor $J_{\mathrm{QSs}}{ }^{*}(\cdot) \leqslant J_{\mathrm{Rss}}{ }^{*}(\cdot)$ holds true.

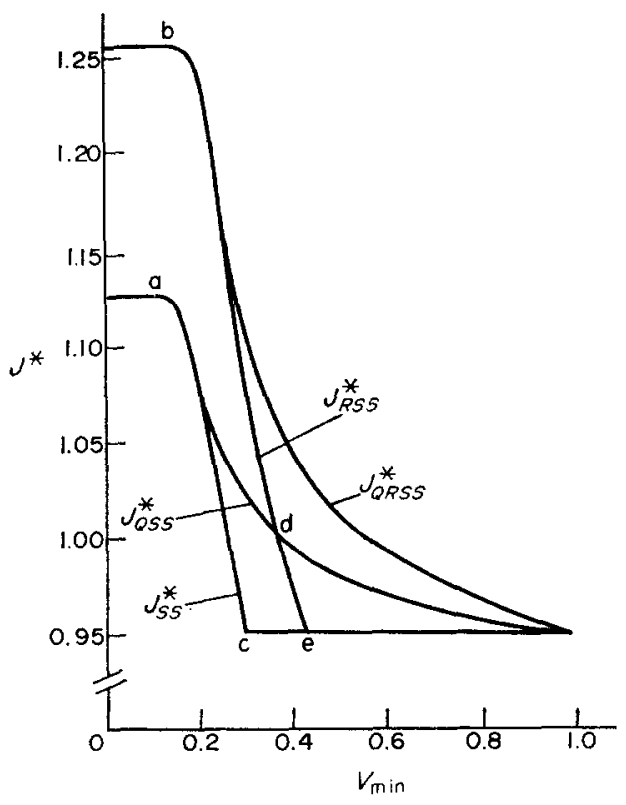

FIG. 2. Optimal cost functions for Example 1 .

\section{Example 2}

The drag and fuel-consumption functions are given by

$$
D(V)=\left\{\begin{array}{l}
0.25 V+1.25 V^{2}-0.5 V^{3}, \quad 0 \leqslant V \leqslant 1 \\
-0.25 V+1.25 V, \quad 1 \leqslant V,
\end{array}\right.
$$

and

$$
F(T)=0 \cdot 1+D^{-1}(T)+0 \cdot 3\left(D^{-1}(T)\right)^{2},
$$

where $D^{-1}(T)$ is the inverse function corresponding to $D(\cdot)$. Since $D(1)=1, V_{\max }=1$. Clearly,

$$
f_{\mathrm{Ss}}(V)=0 \cdot 1+V+0 \cdot 3 V^{2} \text {. }
$$


It can be shown that $D^{-1}(T) \geqslant W(T)$ where

$$
W(T)=\left\{\begin{array}{l}
\frac{4}{3} T, \quad 0 \leqslant T \leqslant \frac{3}{8}, \\
t+\frac{4}{5} T, \quad \frac{3}{8} \leqslant T \leqslant 1 .
\end{array}\right.
$$

Using this inequality it follows that $F(T)>0 \cdot 1+$ $1.3 T$ for $0<T<1$. Thus $(\operatorname{conv} F)(T)=0 \cdot 1+1 \cdot 3 T$ and

$$
f_{\mathrm{RSS}}(V)=0 \cdot 1+1 \cdot 3 D(V) .
$$

Hence $f_{\mathrm{RSS}}(V)<f_{\mathrm{SS}}(V)$ for $0<V<1$. Since $f_{\mathrm{SS}}(\cdot)$ is convex

$$
f_{\mathrm{QSS}}(V)=f_{\mathrm{SS}}(V)
$$

Because the second derivative of $D(\cdot)$ changes sign in $[0,1], f_{\mathrm{RSS}}(\cdot)$ is not convex and this leads to

$$
f_{\mathrm{QRSS}}(V)=\left\{\begin{array}{c}
f_{\mathrm{RSS}}(V), \quad 0 \leqslant V \leqslant 0.75, \\
1 \cdot 4+1 \cdot 665625(V-1)<f_{\mathrm{RSS}}(V), \\
0.75<V<1 .
\end{array}\right.
$$

These results yield the following expressions:

$$
\begin{gathered}
J_{\mathrm{SS}} *(V)=J_{\mathrm{QSS}}{ }^{*}(V)=\left\{\begin{array}{c}
(0 \cdot 7427 \ldots), \\
0 \leqslant V \leqslant 3^{-0.5}, \\
V\left(f_{\mathrm{SS}}(V)\right)^{-1}, \\
3^{-0.5} \leqslant V \leqslant 1,
\end{array}\right. \\
J_{\mathrm{RSS}}{ }^{*}(V)=\left\{\begin{array}{c}
(0 \cdot 9206 \ldots), \quad 0 \leqslant V \leqslant(0 \cdot 2818 \ldots), \\
V\left(f_{\mathrm{RSS}}(V)\right)^{-1}, \quad(0 \cdot 2818 \ldots) \leqslant V \leqslant 1,
\end{array}\right. \\
J_{\mathrm{QRSS}} *(V)=\left\{\begin{array}{c}
J_{\mathrm{RSS}}{ }^{*}(V), \quad 0 \leqslant V \leqslant 0 \cdot 75, \\
V\left(f_{\mathrm{QRSS}}(V)\right)^{-1}, \\
0 \cdot 75 \leqslant V \leqslant 1 .
\end{array}\right.
\end{gathered}
$$

Thus

$$
\begin{gathered}
J_{\mathrm{Ss}} *(V)=J_{\mathrm{QSs}} *(V)<J_{\mathrm{RSs}} *(V)=J_{\mathrm{QRSS}} *(V) \\
\text { for } \quad V \in[0,0 \cdot 75]
\end{gathered}
$$

and

$$
\begin{gathered}
J_{\mathrm{SS}} *(V)=J_{\mathrm{QSS}} *(V)<J_{\mathrm{RSs}}{ }^{*}(V)<J_{\mathrm{QRSS}}{ }^{*}(V) \\
\text { for } V \in(0.75,1) .
\end{gathered}
$$

Therefore RSS control, i.e. 'high' frequency switching between $T=1$ and $T=0$, gives better results than SS control for all $0<V_{\min }<1$. Even though QSS control offers no improvement over SS control, QRSS control, i.e. 'low' frequency switching between $T=1$ and a relaxed $\mathrm{T}(t)$ ('high' frequency switching between $T_{1}=1$ and $T_{2}=0$ with $\lambda_{\mathbf{R}}=D(0.75)$ ), provides an improvement over RSS control.

\section{SOME GENERAL PROPERTIES OF THE OPTIMAL COST FUNCTIONS}

In this section general properties of the functions

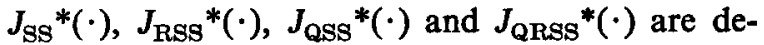
rived. Some of these properties depend on additional assumptions concerning $D(\cdot)$ and $F(\cdot)$ and give information about the possibility (or impossibility) of performance improvement by means of RSS, QSS and QRSS controls.

All four of the SS problems are of the form

$$
J_{A}=V\left(f_{A}(V)\right)^{-1}, \quad V_{\min } \leqslant V \leqslant V_{\max }
$$

with $J_{A}{ }^{*}(\cdot)$ defined by

$$
\begin{gathered}
J_{A}^{*}\left(V_{\min }\right)=\max V\left(f_{A}(V)\right)^{-1} \quad \text { subject to } \\
V \in\left[V_{\min }, V_{\max }\right] .
\end{gathered}
$$

Obviously, $J_{A}{ }^{*}(\cdot)$ is a non-increasing function on $\left[0, V_{\max }\right]$. Lower semi-continuity of $f_{A}(\cdot)$ implies upper semi-continuity of $J_{A}^{*}(\cdot)$. Continuity of $f_{A}(\cdot)$ implies continuity of $J_{A}^{*}(\cdot)$, but the converse is not true. Clearly $J_{\mathrm{RSs}}{ }^{*}(\cdot), J_{\mathrm{QSs}}{ }^{*}(\cdot)$ and $J_{\mathrm{ORSS}}{ }^{*}(\cdot)$ are all continuous.

To obtain additional properties of $J_{A}{ }^{*}(\cdot)$ define

$$
\begin{aligned}
& \hat{V}_{A}=\max V \text { subject to } V\left(f_{A}(V)\right)^{-1} \\
& =J_{A}^{*}(0) \text {. }
\end{aligned}
$$

The maximum exists because the upper semicontinuity of $V\left(f_{A}(V)\right)^{-1}$ implies that the set corresponding to $V\left(f_{A}(V)\right)^{-1}=J_{A}^{*}(0)$ is closed. Clearly

$$
\begin{aligned}
J_{A}^{*}(V)=J_{A}^{*}(0), & 0 \leqslant V \leqslant \hat{V}_{A} \\
& <J_{A}^{*}(0), \quad \hat{\nabla}_{A}<V<V_{\max } .
\end{aligned}
$$

With an additional assumption on $f_{A}(\cdot)$ more can be said.

\section{Theorem 1}

Let $f_{A}(\cdot)$ be convex on $\left[\hat{V}_{A}, V_{\max }\right]$. Then $J_{A}^{*}(\cdot)$ is continuous on $\left[0, V_{\max }\right], J_{A}^{*}(\cdot)$ is strictly decreasing on $\left[\hat{V}_{A}, V_{\max }\right]$ and

$$
J_{A}^{*}(V)=V\left(f_{A}(V)\right)^{-1}, \quad \hat{V}_{A} \leqslant V \leqslant V_{\max } .
$$

The proof depends on the following lemma whose proof is straightforward and is therefore omitted.

\section{Lemma \\ Let}

$$
g(V)=a V+b
$$

have the following properties: $f_{A}\left(V_{0}\right)=g\left(V_{0}\right)$ where $V_{0} \in\left(\hat{V}_{A}, V_{\max }\right], f_{A}(\cdot) \geqslant g(\cdot)$. That is, $g(\cdot)$ is a line of support to $f_{A}(\cdot)$ at $V_{0} \in\left(V_{A}, V_{\max }\right]$. Then $a>\left(J_{A}{ }^{*}(0)\right)^{-1}$ and $b<0$.

Proof. Applying the lemma gives

$$
\begin{aligned}
V\left(f_{A}(V)\right)^{-1}-V_{0}\left(f_{A}\left(V_{0}\right)\right)^{-1}= & \left(V f_{A}\left(V_{0}\right)-V_{0} f_{A}(V)\right) \\
& \times\left(f_{A}\left(V_{0}\right) f_{A}(V)\right)^{-1} \\
\leqslant & \left(V\left(a V_{0}+b\right)\right. \\
& \left.-V_{0}(a V+b)\right) \\
& \times\left(f_{A}\left(V_{0}\right) f_{A}(V)\right)^{-1} \\
\leqslant & b\left(V-V_{0}\right)\left(f_{A}\left(V_{0}\right)\right. \\
& \left.\times f_{A}(V)\right)^{-1} \\
0 \leqslant V \leqslant V_{\max } & <0, \quad V>V_{0} .
\end{aligned}
$$


Thus $V\left(f_{A}(V)\right)^{-1}$ is strictly decreasing on $\left(\hat{V}_{A}\right.$, $\left.V_{\max }\right]$. From (42) it then follows that (45) is true and that $J_{A}^{*}(\cdot)$ is continuous because $f_{A}(\cdot)$ is continuous.

\section{Corollary 2}

For $A=\mathrm{QSS}$ and $A=\mathrm{QRSS}$ the results of Theorem 1 are true. Moreover $\hat{V}_{\mathrm{QSS}}=\hat{V}_{\mathrm{SS}}$ and $\hat{V}_{\text {QRSS }}=\hat{V}_{\text {RSS }}$.

Proof. The first results are consequences of the convexity of $f_{\mathrm{QSS}}(\cdot)$ and $f_{\mathrm{QRSS}}(\cdot)$. The geometric interpretation of $J_{A}^{*}(0)$ is that $V\left(J_{A}^{*}(0)\right)^{-1}$ is a line of support of $f_{A}(\cdot)$ as indicated at the end of Section 2. But $\left(\operatorname{conv} f_{\mathrm{ss}}\right)(\cdot)=f_{\mathrm{QSs}}(\cdot)$ and $f_{\mathrm{Ss}}(\cdot)$ have identical lines of support [5]. Thus $J_{\mathrm{SS}}{ }^{*}(0)=J_{\mathrm{QSS}}{ }^{*}(0)$ and clearly $\hat{V}_{\mathrm{QSS}} \geqslant V_{\mathrm{SS}}$. But $\hat{V}_{\mathrm{QSS}}>\hat{\nabla}_{\mathrm{SS}}$ implies

$$
V\left(f_{\mathrm{QSS}}(V)\right)^{-1}=V\left(f_{\mathrm{Sg}}(V)\right)^{-1} \text { for } V=\hat{V}_{\mathrm{QSS}}
$$

which contradicts the definition of $\hat{V}_{\mathrm{SS}}$. Therefore $\hat{V}_{\text {QSS }}=V_{\text {SS }}$. The identical argument applies to $\hat{V}_{\mathrm{QRSS}}=\hat{V}_{\mathrm{RSS}} \cdot$

\section{Remark 1}

Note that

$$
J_{\mathrm{QRSS}}{ }^{*}(0)=J_{\mathrm{RSS}}{ }^{*}(0) \geqslant J_{\mathrm{QSS}}{ }^{*}(0)=J_{\mathrm{SS}}{ }^{*}(0) .
$$

Thus if $V_{\mathrm{avg}}$ is not constrained $\left(V_{\min }=0\right)$ low frequency switching produces no improvement. This is in agreement with [6].

Under what circumstances does RSS control produce an improvement? The following theorem seems to be about the most that can be said.

\section{Theorem 3}

Let

$$
\begin{aligned}
Q=\left\{V: J_{\mathrm{SS}}{ }^{*}(V)\right. & \\
& \left.=V\left(f_{\mathrm{SS}}(V)\right)^{-1}, V \in\left[0, V_{\max }\right]\right\} .
\end{aligned}
$$

(i) If (conv $F)(T)<F(T)$ for some $T \in D(\mathrm{Q})$ then $J_{\mathrm{Rss}}{ }^{*}(V)>J_{\mathrm{Ss}}{ }^{*}(V)$ for some $V \in\left[0, V_{\max }\right]$. (ii) If $J_{\mathrm{RSS}}{ }^{*}(V)>J_{\mathrm{SS}}{ }^{*}(V)$ for some $V \in\left[0, V_{\max }\right]$ then $(\operatorname{conv} F)(T)<F(T)$ for some $T \in[0,1]$.

Proof. Result (ii) is obvious. Result (i) follows because for $V$ such that $D(V)=T, V\left(f_{\mathrm{Rss}}(V)\right)^{-1}>$ $J_{\mathrm{Ss}}{ }^{*}(V)$.

\section{Remark 2}

It is easy to construct examples which demonstrate the gap between (i) and (ii). Consider, for example, $\quad V\left(f_{\mathrm{sS}}(V)\right)^{-1}<V_{2}\left(f_{\mathrm{Ss}}\left(V_{2}\right)\right)^{-1}$ for all $V \in\left(V_{1}, V_{2}\right), \quad 0 \leqslant V_{1}<V_{2} \leqslant V_{\max }$, and $f_{\mathrm{RgS}}(\cdot)$ such that $f_{\mathrm{RSs}}(V)<f_{\mathrm{sg}}(V)$ for some $V \in\left(V_{1}, V_{2}\right)$ and $V\left(f_{\mathrm{RSS}}(V)\right)^{-1} \leqslant V_{2}\left(f_{\mathrm{SS}}\left(V_{2}\right)\right)^{-1}$ for all $V \in\left[V_{1}, V_{2}\right]$.

\section{Remark 3}

If $F(\cdot)$ is convex on $[0,1]$ then $J_{\mathrm{RSS}}{ }^{*}(\cdot)=J_{\mathrm{SS}}{ }^{*}(\cdot)$ and $J_{\mathrm{QRSS}}{ }^{*}(\cdot)=J_{\mathrm{QSS}} *(\cdot)$.

In the case of QSS control and QRSS control both necessary and sufficient conditions are available for improvement (or non-improvement).

\section{Theorem 4}

$J_{\mathrm{QSS}}{ }^{*}(\cdot)=J_{\mathrm{SS}}{ }^{*}(\cdot)\left(J_{\mathrm{QRSS}}{ }^{*}(\cdot)=J_{\mathrm{RSS}}{ }^{*}(\cdot)\right)$ if and only if $f_{\mathrm{SS}}(\cdot)$ is convex on $\left[\hat{P}_{\mathrm{SS}}, V_{\max }\right]\left(f_{\mathrm{RsS}}(\cdot)\right.$ is convex on $\left[\hat{V}_{\mathrm{RSS}}, V_{\max }\right]$ ).

Proof. With an appropriate change in notation the two cases have the same proof so consider only the QSS case. If $f_{\mathrm{SS}}(\cdot)$ is convex on $\left[\hat{V}_{\mathrm{SS}}, V_{\max }\right]$ then $f_{\mathrm{Ss}}(\cdot) \geqslant h(\cdot)$ where $h(V)=\left(J_{\mathrm{SS}}{ }^{*}(0)\right)^{-1} V$ for $0 \leqslant V \leqslant \hat{V}_{\mathrm{SS}},=f_{\mathrm{SS}}(V)$ for $\hat{V}_{\mathrm{SS}} \leqslant V \leqslant V_{\max }$. Since $h(\cdot)$ is convex $f_{\mathrm{SS}}(\cdot) \geqslant\left(\operatorname{conv} f_{\mathrm{SS}}\right)(\cdot) \geqslant h(\cdot)$. Thus $f_{\mathrm{SS}}(\cdot)=\left(\operatorname{conv} f_{\mathrm{SS}}\right)(\cdot)$ on $\left[\tilde{V}_{\mathrm{SS}}, V_{\max }\right]$ and because of Corollary $2, \quad J_{\mathrm{QSS}} *(\cdot)=J_{\mathrm{SS}} *(\cdot)$. Now suppose $J_{\mathrm{QSS}}{ }^{*}(\cdot)=J_{\mathrm{SS}}{ }^{*}(\cdot)$ and $f_{\mathrm{Ss}}(\cdot)$ is not convex on $\left[\hat{\mathrm{SS}}_{\mathrm{SS}}, V_{\mathrm{max}}\right]$. Then there is a line of support to $f_{\mathrm{SS}}(\cdot)$ which contacts $f_{\mathrm{SS}}(\cdot)$ at $V_{1}$ and $V_{2}$, $\hat{V}_{\mathrm{SS}} \leqslant V_{1}<V_{2} \leqslant V_{\max }$ and does not contact $f_{\mathrm{SS}}(\cdot)$ on $\left(V_{1}, V_{2}\right)$, i.e. $g\left(V_{1}\right)=f_{\mathrm{SS}}\left(V_{\mathrm{i}}\right), \mathrm{i}=1,2$ and $g(V)=f_{\mathrm{SS}}(V)$ for $V \in\left(V_{1}, V_{2}\right)$. Moreover, $g(V)=f_{\mathrm{QSS}}(V)$ for $V \in\left[V_{1}, V_{2}\right]$ and $g(V) \leqslant f_{\mathrm{Ss}}(V)$ for $V \in\left[0, V_{\max }\right]$. Take $V_{\min } \in\left(V_{1}, V_{2}\right)$. Because $J_{\mathrm{QSS}}{ }^{*}(\cdot)=J_{\mathrm{SS}}{ }^{*}(\cdot)$

$$
V\left(f_{\mathrm{SS}}(V)\right)^{-1}=J_{\mathrm{QSS}} *\left(V_{\min }\right)
$$

has a solution for some $V \in\left[V_{\min }, V_{\max }\right]$. Using $g(\cdot) \leqslant f_{\mathrm{ss}}(\cdot)$ this means that the system

$$
\begin{array}{r}
V(a V+b)^{-1} \leqslant V_{\min }\left(a V_{\min }+b\right)^{-1}, \\
V \in\left[V_{\min }, V_{\max }\right]
\end{array}
$$

has a solution. But this can be written

$$
0 \leqslant b\left(V-V_{\min }\right), V \in\left[V_{\min }, V_{\max }\right] .
$$

Since by the lemma $b<0, V=V_{\min }$. Substituting into (49), $f_{\mathrm{SS}}\left(V_{\min }\right)=f_{\mathrm{QSS}}\left(V_{\min }\right)$ which contradicts $f_{\mathrm{QSS}}(V)=g(V)<f_{\mathrm{SS}}(V)$ for $V \in\left(V_{1}, V_{2}\right)$.

\section{Remark 4}

If $f_{\mathrm{SS}}(\cdot)$ has a discontinuity on $\left[\hat{V}_{\mathrm{SS}}, V_{\mathrm{max}}\right], f_{\mathrm{SS}}(\cdot)$ is not convex on $\left[\hat{V}_{\mathrm{SS}}, V_{\mathrm{max}}\right]$. Thus $J_{\mathrm{QSs}} *(V)>$ $J_{\mathrm{Ss}}{ }^{*}(V)$ for some $V \in\left[0, V_{\max }\right]$.

\section{Corollary 5}

If $F(\cdot)$ is convex on $[0,1]$ and $D(\cdot)$ is convex on $\left[\hat{V}_{\mathrm{SS}}, V_{\max }\right]$ then

$$
J_{\mathrm{QRSS}}{ }^{*}(\cdot)=J_{\mathrm{QSS}}{ }^{*}(\cdot)=J_{\mathrm{RSS}}{ }^{*}(\cdot)=J_{\mathrm{SS}}{ }^{*}(\cdot) \text {. }
$$

Proof. The first and last equalities follow from Remark 3. Since both $F(\cdot)$ and $D(\cdot)$ are convex and $F(\cdot)$ is non-decreasing it turns out [5] that $f_{\mathrm{ss}}(V)=F(D(V))$ is convex on $\left[\vec{P}_{\mathrm{Ss}}, V_{\max }\right]$. Thus the theorem gives middle equality. 


\section{Corollary 6}

If $D(\cdot)$ is convex on $\left[\hat{V}_{\mathrm{RSS}}, V_{\max }\right]$ then

$$
J_{\mathrm{QRSS}}{ }^{*}(\cdot)=J_{\mathrm{RSS}}{ }^{*}(\cdot) \text {. }
$$

Proof. $f_{\mathrm{Rss}}(\cdot)$ is a non-decreasing convex function of a convex function and is therefore convex on $\left[\hat{V}_{\mathrm{RSS}}, V_{\max }\right]$. The theorem applies immediately.

\section{Remark 5}

There are examples which show that $J_{Q S S}{ }^{*}(V)>$ $J_{\mathrm{SS}}{ }^{*}(V)$ and $J_{\mathrm{RSS}}{ }^{*}(V)>J_{\mathrm{SS}} *(V)$ are both possible when $D(\cdot)$ is convex. Thus it is not possible to draw a stronger conclusion that Corollary 6 .

There is another conclusion which can be made about the lack of superiority of $J_{\mathrm{QRSS}}{ }^{*}(\cdot)$.

\section{Theorem 7}

$$
\text { If } J_{\mathrm{QSS}} *(\cdot) \geqslant J_{\mathrm{RsS}}{ }^{*}(\cdot) \text { then } J_{\mathrm{QRSS}}{ }^{*}(\cdot)=J_{\mathrm{QSS}}{ }^{*}(\cdot) \text {. }
$$

Proof. By Corollary 2 and the definition of $J_{\mathrm{RSS}}{ }^{*}(\cdot), \quad V\left(f_{\mathrm{RSS}}(V)\right)^{-1} \leqslant V_{\min }\left(f_{\mathrm{QSS}}\left(V_{\min }\right)\right)^{-1}$ for all $V \geqslant V_{\min } \geqslant \hat{V}_{\mathrm{sS}}$. Thus $f_{\mathrm{Rss}}(V) \geqslant f_{\mathrm{QSS}}(V)$ for $V \in\left[\mathbb{P}_{\mathrm{SS}}, V_{\max }\right]$. Also $f_{\mathrm{RSS}}(V) \geqslant\left(J_{\mathrm{QSs}}{ }^{*}(0)\right)^{-1} V$ for $V \in\left[0, \hat{V}_{\mathrm{SS}}\right]$. Let $f_{A}(V)=f_{\mathrm{QSS}}(V)$ for $V \in\left[\hat{V}_{\mathrm{SS}}, V_{\mathrm{max}}\right]$, $=\left(J_{\mathrm{QSS}} *(0)\right)^{-1} V$ for $V \in\left[0, \hat{V}_{\mathrm{Ss}}\right]$. Since $f_{A}(\cdot)$ is convex and $f_{\mathrm{RSS}}(\cdot) \geqslant f_{A}(\cdot), f_{\mathrm{QRSS}}(\cdot)=\left(\operatorname{conv} f_{\mathrm{RSS}}\right)$ $(\cdot) \geqslant f_{\mathrm{A}}(\cdot)$. But $J_{\boldsymbol{A}}{ }^{*}(\cdot)=J_{\mathrm{QSS}}{ }^{*}(\cdot)$ and hence $J_{\mathrm{QRSS}}{ }^{*}(\cdot) \leqslant J_{A}{ }^{*}(\cdot)=J_{\mathrm{QSS}}{ }^{*}(\cdot)$. Since $J_{\mathrm{QRSS}}{ }^{*}(\cdot) \geqslant$ $J_{\mathrm{QSS}}{ }^{*}(\cdot)$ the proof is complete.

\section{Remark 6}

Theorem 7 shows that QRSS control can be better than both RSS and QSS control only if $J_{\mathrm{QSS}}{ }^{*}(\cdot) \geqslant J_{\mathrm{RsS}}{ }^{*}(\cdot)$ is not true, i.e. $J_{\mathrm{QSs}}{ }^{*}(V)<$ $J_{\mathrm{RSs}}{ }^{*}(V)$ for some $V \in\left[0, V_{\max }\right]$. Examples 1 and 2 of the previous section demonstrate this requirement. In Example 1 neither $J_{\mathrm{QSs}}{ }^{*}(\cdot) \geqslant J_{\mathrm{Rss}}{ }^{*}(\cdot)$ nor $J_{\mathrm{RSs}} *(\cdot) \geqslant J_{\mathrm{QSs}}{ }^{*}(\cdot)$ holds. In Example 2 $J_{\mathrm{RSS}}{ }^{*}(\cdot) \geqslant J_{\mathrm{QSS}}{ }^{*}(\cdot)$ and in addition $J_{\mathrm{QSS}}{ }^{*}(\cdot)=$ $J_{\mathrm{SS}}{ }^{*}(\cdot)$. It is not difficult to construct examples where $J_{\mathrm{RSS}}{ }^{*}(\cdot) \geqslant J_{\mathrm{QSS}}{ }^{*}(\cdot)$ and $J_{\mathrm{QSS}}{ }^{*}(\cdot) \neq J_{\mathrm{SS}}{ }^{*}(\cdot)$. Thus all the possibilities of $J_{\mathrm{QRss}}{ }^{*}(V)>J_{\mathrm{RSs}}{ }^{*}(V)$ and $J_{\text {QRSs }} *(V)>J_{\text {QSs }}{ }^{*}(V)$ allowed by Theorem 7 do in fact exist.

\section{OTHER PERIODIC CONTROLS, SOLUTION OF OPC}

In Section $2 J^{*}\left(V_{\min }\right)$ was defined as the supremum of (1) subject to constraints (2-6) and it was noted that $J^{*}(\cdot) \geqslant J_{\text {QRSS }} *(\cdot) \geqslant J_{\text {RSs }} *(\cdot)$, $J_{\mathrm{QSS}}{ }^{*}(\cdot) \geqslant J_{\mathrm{Ss}} *(\cdot)$. This raises two questions. (i) Do there exist periodic controls which perform better than QRSS controls $\left(J^{*}(V)>J_{\mathrm{QRSS}}{ }^{*}(V)\right.$ for some $\left.V \in\left[0, V_{\max }\right]\right)$ ? (ii) Does OPC have a solution $\left(J^{*}\left(V_{\min }\right)=\max J(T(\cdot), V(\cdot), \tau)\right)$ ? In general, question (ii) must be answered negatively. It is easy to give examples where $J^{*}(V)=J_{\mathrm{Rss}}{ }^{*}(V)$ or $J^{*}(V)=J_{\mathrm{QSS}} *(V)$ and there is no triple $(T(\cdot)$, $V(\cdot), \tau)$ which satisfies (2-6) and achieves the supremum.

To answer question (i), at least in part, an upper bound for $J(T(\cdot), V(\cdot), \tau)$ will be derived. Define

$$
D_{\mathrm{avg}}=\frac{1}{\tau} \int_{0}^{\tau} D(V(t)) \mathrm{d} t
$$

and note from (2) that $D_{\mathrm{avg}}=T_{\mathrm{avg}}$. Write $V(t)=V_{\mathrm{avg}}+\Delta V(t)$ and observe that

$$
\begin{aligned}
D(V(t)) & \geqslant(\operatorname{conv} D)\left(V_{\mathrm{avg}}+\Delta V(t)\right) \\
& \geqslant(\operatorname{conv} D)\left(V_{\mathrm{avg}}\right)+q \Delta V(t)
\end{aligned}
$$

where $q$ is the slope of a line of support to (conv $D)(\cdot)$ at $V_{\text {avg. }}$. By definition the average of $\Delta V(t)$ over the interval $[0, \tau]$ is zero and thus

$$
\begin{aligned}
D_{\mathrm{avg}} & \geqslant \frac{1}{\tau} \int_{0}^{\tau}\left[(\operatorname{conv} D)\left(V_{\mathrm{avg}}\right)+q \Delta V(t)\right] \mathrm{d} t \\
& \geqslant(\operatorname{conv} D)\left(V_{\mathrm{avg}}\right) .
\end{aligned}
$$

In the same manner it follows that

$$
F_{\text {avg }} \geqslant(\operatorname{conv} F)\left(T_{\text {avg }}\right) \text {. }
$$

Using (53), (54), $T_{\mathrm{avg}}=D_{\mathrm{avg}}$, and the fact that (conv $F)(\cdot)$ is a non-decreasing function gives $F_{\mathrm{avg}} \geqslant(\operatorname{conv} F)\left((\operatorname{conv} D)\left(V_{\mathrm{avg}}\right)\right)$. This yields the desired upper bound:

$$
\begin{aligned}
J(V(\cdot), T(\cdot), \tau) & \leqslant[(\operatorname{conv} F)((\operatorname{conv} D) \\
& \left.\left.\times\left(V_{\mathrm{avg}}\right)\right)\right]^{-1} V_{\mathrm{avg}} .
\end{aligned}
$$

If $D(\cdot)$ is convex (55) gives

$$
\begin{aligned}
J^{*}\left(V_{\min }\right)= & \sup J(V(\cdot), T(\cdot), \tau) \\
\leqslant & \max V_{\text {avg }}\left(f_{\mathrm{RSS}}\left(V_{\mathrm{avg}}\right)\right)^{-1} \\
& \quad \text { subject to } V_{\text {avg }} \in\left[V_{\min }, V_{\mathrm{max}}\right] \\
\leqslant & J_{\mathrm{RSS}} *\left(V_{\min }\right) .
\end{aligned}
$$

Since $J_{\mathrm{RSS}} *\left(V_{\min }\right) \leqslant J^{*}\left(V_{\min }\right)$ this proves the following.

\section{Theorem 8.}

If $D(\cdot)$ is convex $J^{*}(\cdot)=J_{\mathrm{RSs}}{ }^{*}(\cdot)$.

\section{Remark 7.}

When $D(\cdot)$ is convex $J^{*}(\cdot)=J_{\mathrm{QRSS}} *(\cdot)=$ $J_{\mathrm{RSS}}{ }^{*}(\cdot) \geqslant J_{\mathrm{QSS}} *(\cdot) \geqslant J_{\mathrm{SS}}{ }^{*}(\cdot)$ (see Corollary 6 ). Thus there is no triple $(V(\cdot), T(\cdot), \tau)$ which does better than RSS control. 
Remark 8.

When $D(\cdot)$ and $F(\cdot)$ are convex

$J^{*}(\cdot)=J_{\mathrm{QRSS}} *(\cdot)=J_{\mathrm{RSS}} *(\cdot)=J_{\mathrm{QSS}} *(\cdot)=J_{\mathrm{SS}} *(\cdot)$

per Corollary 5. Thus the SS control is a solution of OPC.

In searching through many examples it has not been possible to show that the following is false.

\section{Conjecture}

$$
J *(\cdot)=J_{\mathrm{QRSS}} *(\cdot) .
$$

This conjecture answers questions (i) negatively with no additional assumptions on $D$ and $F$. If the conjecture is true it is a strong result which depends on the special structure of the OPC problem stated in Section 1. There is no reason to expect that it would hold in more complex vehicle cruise problems.

\section{CONCLUSIONS, POSSIBLE APPLICATIONS}

A simple model (1-6) for optimizing vehicle cruise has been presented and it has been shown that QRSS, RSS and QSS control may all produce better results than conventional SS control. The potential advantage of QRSS control has been demonstrated, although such advantage requires that both $D(\cdot)$ and $F(\cdot)$ be non-convex as stated in Remark 3 and Corollary 6. Furthermore, if both $D(\cdot)$ and $F(\cdot)$ are convex all modes of steady-state control are equivalent to conventional SS control, per Corollary 5, which is a solution of OPC as indicated in Remark 8.

The simple model is probably not an accurate description of most practical vehicle cruise problems, but it is sufficiently good to suggest practical applications. Consider, for instance, Fig. 3 , which shows fuel-flow rate vs speed for a typical model of a gas turbine ship [7]. Although the dynamic model of the ship is much more complex than (2), $f(V)$ plays the same role as $f_{\mathrm{SS}}(V)$ in this paper. Thus for $V_{\min } \leqslant V_{1}$ maximum specific range (steady-state conditions) is obtained at $V=V_{1}$ with one engine operating. However, for $V_{\min } \in\left(V_{2}\right.$, $V_{3}$ ) quasi-steady-state operation produces better fuel economy than two-engine steady-state operation as implied by Remark 4. For the model in [7] the difference may be as much as $16 \%$. The quasisteady-state mode requires cycling between the speed $V_{2}$ (one engine) and the speed $V_{3}$ (two engines), but the period is very long, perhaps hours, and thus the cycling should not be difficult to implement. Another possible application is to aircraft cruise. If an optimal periodic control problem is formulated for the aircraft $(F-4)$ discussed in [8] it is possible to show that relaxed-steady-state control gives improved cruise performance. However, there are questions concerning the accuracy of the airplane model which must be investigated further.

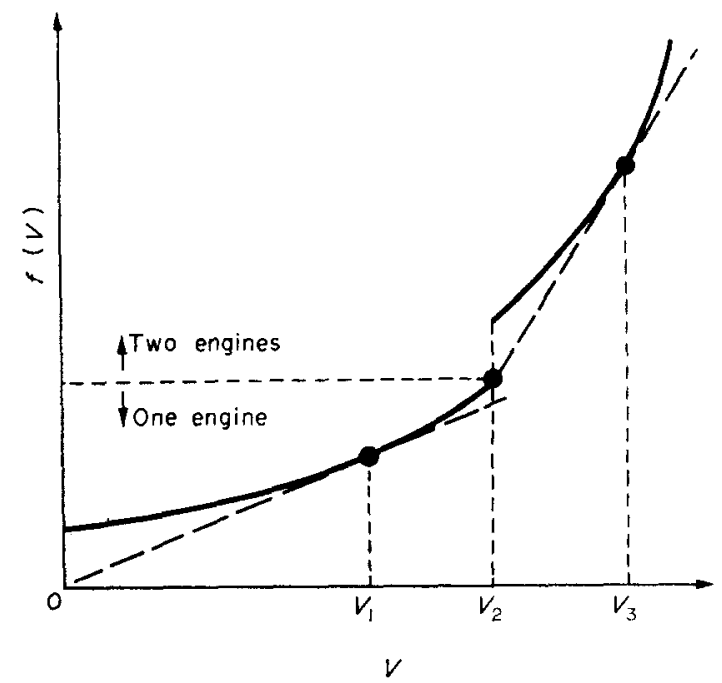

FIG. 3. Steady-state fuel rate for ship.

Acknowledgements-This research is based on work sponsored by the Air Force Office of Scientific Research under Grant No. AFOSR 73-2517. It was carried out while the author was on leave at the Department of Electrical Engineering, The Johns Hopkins University. The helpful comments of staff and students there are appreciated.

\section{REFERENCES}

[1] J. E. BaIley: Periodic operation of chemical reactors, a review. Chem. Engng Commun. 1, 111-124 (1973).

[2] G. Guardabassi, A. Locatelli and S. Rinaldi: Status of periodic optimization of dynamical systems. J. Opt. Theory Appl. 14, 1-20 (1974).

[3] E. G. Gilbert: Optimal periodic control, a general theory of necessary conditions (in preparation).

[4] J. E. BAILEY: Necessary conditions for optimality in a general class of nonlinear mixed boundary value control problems. Int. J. Control 16, 311-320 (1972).

[5] R. T. Rockafellar: Convex Analysis. Princeton University Press (1970).

[6] A. LOCATELLI and S. RINALDI: Optimal quasi-stationary periodic processes. Automatica 6, 779-785 (1970).

[7] C. J. RUBIS: Acceleration and steady-state propulsion dynamics of a gas turbine ship with controllable-pitch propeller. Trans. Soc. Naval Arch. Marine Engng 8, 329-360 (1972).

[8] N. R. Zagalsky, R. P. Irons and R. L. Schultz: Energy state approximation and minimum-fuel fixedrange trajectories. J. Aircraft 8, 488-490 (1971). 